\title{
Anaesthesia for diagnostic muscle biopsy in an infant with Pompe's disease
}

The anaesthetic management of children with glycogenstorage disease type lla (Pompe's disease) presents a variety of challenges. A modification of a femoral nerve block, the inguinal paravascular block, as described by Winnie, was used in conjunction with intravenous ketamine to provide anaesthesia for a diagnostic muscle biopsy in a 5.5-month-old infant with Pompe's disease. $A$ peripheral nerve stimulator was used to locate the femoral nerve in lieu of eliciting a paraesthesia.

Glycogen-storage disease type IIa, or Pompe's disease, is an unusual disorder of glycogen metabolism in which all muscle types (smooth, striated, and cardiac) are infiltrated by glycogen deposits. ${ }^{1}$ Anaesthesia presents many difficulties for these patients. The large, protruding, glycogen-infiltrated tongue and poor muscle tone predispose them to upper airway obstruction. Their impaired neurologic function is manifested by decreased cough and gag reflexes and incoordination of swallowing. Aspiration and atelectasis are common problems. In

\section{Key words}

METABOLISM: glycogenosis, glycogen-storage disease, Pompe's disease; ANAESTHESIA, CONDUCTION: femoral nerve block; ANAESTHESIA, INTRAVENOUS: ketamine; ANAESTHESIA: paediatric.

Received from the Department of Anesthesiology and Child Health and Development, Children's Hospital National Medical Center and the George Washington University Medical Center, Washington, D.C.

Address correspondence to: Dr. Lynn M. Broadman, Children's Hospital National Medical Center, 111 Michigan Avenue, Northwest, Washington, D.C. 20010. addition, these patients tolerate changes in haemodynamic function poorly.

Only two case reports outlining the anaesthetic management of patients with glycogen storage disease type lla can be found in the literature. ${ }^{2 *}$ Both involve the use of general anaesthesia. General anaesthesia with halothane, nitrous oxide, and oxygen resulted in irreversible cardiac arrest following an uncomplicated induction in one patient $^{2}$ and in laryngospasm during laryngoscopy in another.* Kaplan suggests that a caudal peridural block may be the anaesthetic of choice for the performance of diagnostic muscle biopsies in the lower extremities in patients suspected of having Pompe's disease.* This suggestion, however, is based on theoretical considerations; no one has reported the use of a caudal block or any other regional anaesthetic technique for this purpose.

\section{Case report}

A 5.5-month-old, $6.4 \mathrm{~kg}$ female infant was admitted to the hospital with hypotonia and congestive heart failure one week prior to muscle biopsy surgery. Her past medical history was otherwise negative. The family history was positive for myotonia congenita in a distant cousin. The patient's only medication was spironolactone $(3 \mathrm{mg}$ PO bid), begun one week prior to surgery, for the control of congestive heart failure. Although somewhat floppy, the infant was in no distress. The child had a large tongue. Physical examination revealed slight hepatomegaly with the liver border situated $1-2 \mathrm{~cm}$ below the costal margin. The apical impulse was palpated at the left axillary line. Auscultation revealed a regular rate and rhythm with an S4 gallop. A II/VI systolic ejection murmur was noted

${ }^{*}$ Kaplan $R$. Pompe's disease presenting for anesthesia. Anesthesiology Review 1980; 7: 21-8. 
along the left sternal border. The lungs were clear. The chest $x$-ray revealed massive cardiomegaly, with the cardiac silhouette filling the entire left hemithorax. In addition, the left lower lobe was totally collapsed. A cardiac consultation was obtained. The electrocardiogram showed both biatrial and biventricular hypertrophy and a shortened P-R interval (0.09). Echocardiography revealed massive biventricular hypertrophy with obliteration of the left ventricular cavity, decreased contractility, and paradoxical septal wall motion; however, neither left nor right ventricular outflow tract obstruction was detected.

These findings indicated a hypertrophic cadiomyopathy, most probably Pompe's disease. A diagnostic muscle biopsy was scheduled to obtain histologic evidence of a glycogen-storage disease. The patient was made NPO after midnight and this status was maintained for 11 hours prior to the induction of anaesthesia. An intravenous infusion of 0.3 normal saline in five per cent dextrose was started at midnight at a rate of $24 \mathrm{ml} \cdot \mathrm{hr}^{-1}$. No premedication was given. A blood pressure cuff with doppler, EKG, axillary temperature probe, and precordial stethoscope were applied for intraoperative monitoring. The vital signs initially obtained in the operating room were: blood pressure $90 \mathrm{mmHg}$ systolic, heart rate 135 , respiratory rate 45. The infant was irritable and crying. Ketamine $\left(0.5 \mathrm{mg} \cdot \mathrm{kg}^{-1}\right)$ was administered intravenously for sedation. Glycopyrrolate $(0.05 \mathrm{mg})$ was given in conjunction with the ketamine as an antisialagogue. After five minutes the infant remained restless. There was no change in the patient's vital signs. The dose of intravenous ketamine was repeated $\left(0.5 \mathrm{mg} \cdot \mathrm{kg}^{-1}\right)$ and the infant became placid. Again, no changes in blood pressure, heart rate, respiratory effort or secretions were noted. A modification of a femoral nerve block, ${ }^{3}$ the inguinal paravascular block, was then performed in the following manner: the child was placed in the supine position. The anterior superior iliac spine and the pubic tubercle were palpated. A line that approximates the course of the inguinal ligament was drawn between the two aforementioned landmarks. Because it was not possible to palpate the femoral artery, a doppler probe was used to locate it. We then used the femoral artery to approximate the location of the femoral nerve, since the nerve lies lateral to the femoral artery as they both pass beneath the inguinal ligament. The skin was painted with povidone-iodine. A peripheral nerve stimulator (Professional Instruments Company, Model NS3A) was used to locate the femoral nerve. The ground electrode was placed on a region of the chest wall that was well removed from the area where the block was to be performed. The stimulating electrode was attached to the metal hub of a 23 gauge 1 -inch needle.

A two $\mathrm{Hz}$ stimulus of two milliamperes intensity was applied every second through the needle to the skin just lateral to the femoral artery. No motor activity could be detected in the quadriceps muscle group. The needle, which was directed slightly cephalad, was then advanced and withdrawn at several different points along the course of the inguinal ligament just lateral to the femoral artery. Each of these points was approximately $1-2 \mathrm{~mm}$ apart. The point of entry and needle depth that produced the most intense motor activity in the quadriceps group was assumed to coincide with a location where the needle tip was in very close proximity to the femoral nerve. ${ }^{4}$ A test dose of $0.25 \mathrm{ml}$ of lidocaine one per cent with epinephrine $1: 200,000$ was injected, and all quadriceps muscle motor activity ceased. A total volume of $3 \mathrm{ml}$ of lidocaine one per cent with epinephrine 1:200,000 was injected while applying digital pressure distal to the point of needle entry in order to promote cephalad spread of the local anaesthetic. Painful stimuli applied to the blocked extremity prior to the onset of surgery demonstrated anaesthesia to the region innervated by the femoral, lateral femoral cutaneous, and obturator nerves, while similar stimuli applied to the contralateral leg produced a grimace on the child's face and withdrawal of the leg. It was not necessary to restrain the infant at any time during surgery. The child slept throughout most of the procedure; however, she was easily arousable with tactile stimuli.

The patient did not respond to the skin incision, which occurred 25 minutes after the second dose of ketamine, and no change in her vital signs could be detected. The head and upper chest of the infant were visible to the anaesthetist. The patient remained pink throughout the surgery and no change in respiratory effort was detected. The infant became restless 20 minutes after the start of surgery and began to move her other limbs. A third intravenous dose of ketamine $0.5 \mathrm{mg} \cdot \mathrm{kg}^{-1}$ prompt- 
ly controlled this behaviour. Again, no changes in her vital signs were observed. The total dosage of ketamine was $9.5 \mathrm{mg}\left(1.5 \mathrm{mg} \cdot \mathrm{kg}^{-1}\right)$. The patient was transferred to the recovery room 15 minutes after the last dose of ketamine. She appeared awake and alert. A good sucking response was present. Her vital signs were: heart rate 132 , respiratory rate 48 , systolic blood pressure 80 . She resumed her oral intake on the afternoon of the day of surgery and was discharged on the first postoperative day. The diagnosis of Pompe's disease was confirmed by the histologic specimen.

\section{Discussion}

Glycogen-storage disease Cori type IIa is a result of the absence of the enzyme alpha 1, 4 glucosidase (acid maltase). ${ }^{5}$ Glycogen is deposited in many organs: heart, skeletal muscle, smooth muscle, brain, spinal cord, kidney, liver, spleen, and tongue ${ }^{5.6}$ In patients with Pompe's disease, myocardial involvement is the most prominent feature. Infants with this condition, although often normal in appearance at birth, usually develop signs of congestive heart failure by two to three months of age and die within the first year of life. The clinical presentation is characterized by hypotonia and congestive heart failure. The loss of muscle tone is manifest by a weak sucking reflex, poor feeding, hyporeflexia, and retrogression of motor skills. These changes progress to failure to thrive and flaccid paralysis. Circumoral cyanosis, sweating, and respiratory distress suggest the development of congestive heart failure. ${ }^{7}$ A heart murmur, if present, is usually insignificant. Typical electrocardiographic changes include a short P-R interval (PR $\leq 0.09 \mathrm{sec}$ ), a wide QRS complex, and left ventricular hypertrophy. ${ }^{8}$ The cardiac pathophysiology, which can be demonstrated by echocardiography, is that of ventricular hypertrophy and outflow tract obstruction. ${ }^{9}$ Interventricular septal hypertrophy may be present. The hypertrophic septum may either encroach upon the left ventricular outflow tract, causing subaortic stenosis, or if large enough, may cause narrowing of both ventricular outflow tracts. ${ }^{10}$ Hohn suggests that all patients with Pompe's disease have ventricular hypertrophy, while only one-half of these have sufficient septal hypertrophy to cause outflow interference. Histologic abnormalities of the conduction system have been found and implicated in the development of the short P-R interval. ${ }^{11}$ At autopsy, the heart is usually five to six times the expected normal size. ${ }^{12}$

The goals and objectives in the management of this patient were: to provide satisfactory surgical anaesthesia, minimize changes in cardiopulmonary function, minimize distortion of the biopsy specimen, preserve protective airway reflexes, and avoid aspiration and airway obstruction in the perioperative period.

There are many theoretical contraindications to various anaesthetic techniques in patients with glycogen-storage disease type IIa. Myocardial depressants (halothane, enflurane nitrous oxide, and barbiturates) may produce cardiac decompensation. A decrease in either preload or afterload and/or an increase in heart rate and myocardial contractility may precipitate subaortic stenosis or biventricular outflow tract obstruction. ${ }^{10,13}$,* During cardiac catheterization of a child with Pompe's disease, Hohn showed that the gradient between the left ventricle and the aorta increased from 5 torr to 73 torr when subaortic ouflow tract obstruction was precipitated by stimulating the heart with isoproterenol. ${ }^{10}$ A decrease in both preload and afterload associated with isoflurane, butyrophenones, phenothiazines, and spinal and peridural anaesthesia can produce decompensation by similar mechanisms. Although recently reported as a safe alternative to general anaesthesia in high-risk infants, ${ }^{14}$ spinal anaesthesia can, theoretically, produce hypotension and a reduction in myocardial preload. In a patient with subaortic outflow tract obstruction, the pathophysiology of which is very similar to idiopathic hypertrophic subaortic stenosis, spinal anaesthesia and possibly peridural anaesthesia, may be contraindicated. $^{15}$

Ketamine can cause deleterious effects on heart rate and afterload when used in large doses. Kaplan hypothesizes that its use in patients with Pompe's disease may produce outflow tract obstruction due to its inotropic and chronotropic effects or possibly heart failure by increasing afterload. ${ }^{*}$ However, he used ketamine as his primary anaesthetic in one of his case reports. * Ketamine has been used in a small dose (1-2 $\left.\mathrm{mg} \mathrm{kg}^{-1}\right)$ in a large series of patients with neuromuscular disease without adverse effects. Lees et al. successfully anaesthetized 101 patients

* Kaplan $R$. Pompe's disease presenting for anesthesia. Anesthesiology Review 1980; 7: 21-8. 
with neuromuscular diseases presenting for muscle biopsy with low-dose ketamine used in conjunction with a meperidine, pentobarbitone, and atropine premedication and local infiltration. $\dagger$

Patients with Pompe's disease are at risk for perioperative pulmonary complications because of their enlarged tongues, poor muscle tone, decreased respiratory reserves, and neurologic incoordination. They may be sensitive to the respiratory depressant effects of sedatives and narcotics. Agents that increase secretions or cause airway irritability, such as ketamine or methohexitone, may not be desirable. Depolarizing and nondepolarizing muscle relaxants should be used cautiously in all patients with neuromuscular dysfunction.

Our patient was monitored with a blood pressure cuff and precordial stethoscope. Skin colour and capillary refill were checked at frequent intervals. No supplemental oxygen was given. Oximetry was not available in our institution at that time; however, it would have been a useful supplemental monitoring device. Cimetidine, metoclopromide, or both, could likewise have been used as premedicants to decrease the risk of aspiration.

In order to prevent distortion of the biopsy specimen, local infiltration of the skin and muscle must be avoided. It is for this reason that the caudal approach to peridural anaesthesia has been proposed as the technique of choice for patients with Pompe's disease undergoing muscle biopsy. *However, the use of caudal or other regional anaesthesia for lower extremity muscle biopsy in Pompe's disease has not been reported.

We chose a modification of the femoral nerve block, the inguinal paravascular technique of lumbar plexus blockade as described by Winnie, ${ }^{3}$ as one method that might satisfy all of the goals of an ideal anaesthetic in these patients. This approach eliminates the need to perform separate neuroblockade on either the obturator or the lateral femoral cutaneous nerve of the thigh. Winnie uses the femoral nerve to locate the fascial plane between the psoas major, iliacus, and quadratus lumborum

* Kaplan $R$. Pompe's disease presenting for anesthesia Anesthesiology Review 1980; 7: 21-8.

†Lees DE, Kim YD, Macnamara TE. The safety of ketamine in pediatric neuromuscular disease (a study of 101 patients). Anesthesiology Review 1982; 9: 17-21. muscles. He has shown that when a relatively large volume of local anaesthetic is introduced into the "fascial sandwich" formed by these three muscles, cephalad spread of the drug occurs. The lumbar plexus (obturator, lateral femoral cutaneous, and femoral nerves) lies within this sandwich. It is the cephalad spread of the local anaesthetic that results in neuroblockade of the obturator and lateral femoral cutaneous nerve. To perform the inguinal paravascular block in adults, Winnie entered the femoral perineural envelope lateral to the femoral artery and elicited a paraesthesia. A volume of local anaesthetic equal to $0.5 \mathrm{mg} \cdot \mathrm{kg}^{-1}$ was then injected while distal digital pressure was applied to promote cephalad spread of the agent. We used a peripheral nerve stimulator to locate the femoral nerve as an alterantive to eliciting a paraesthesia in this infant.

There are several potential advantages of using the inguinal paravascular block in lieu of other regional techniques, such as the caudal block suggested by Kaplan.* One sees only minimal alterations in sympathetic or venous tone with the femoral nerve block, and these alterations are limited to the extremity being blocked. Furthermore, with this technique there is no risk of an inadvertent dural puncture and the resultant high spinal anaesthetic. On the other hand, there is always the potential risk of causing nerve, artery, or vein damage when performing neuroblockade. Finally, there is always the risk of adverse systemic reactions when using potentially toxic doses of local anaesthetics. A large volume of local anaesthetic was required in this case in order to ensure a successful block of the three components of the lumbar plexus. This large volume led to the use of a relatively large dose of lidocaine $\left(5 \mathrm{mg} \cdot \mathrm{kg}^{-1}\right)$, which, in tum, necessitated the vasoconstrictive agent epinephrine.

The choice of an ideal local anaesthetic agent for these patients is difficult. Adequate duration of neural blockade must be achieved to allow one to complete the biopsy. The anticipated duration of this surgery was 30 minutes. It was assumed that a lidocaine solution that contained a vasoconstrictor would provide adequate anaesthesia for at least one hour. Myocardial depression associated with local anaesthetic overdosage is always a potential hazard. We did not use bupivacaine in our patient because of its potential cardiotoxicity. One may criticize the use of a local anaesthetic solution containing 
epinephrine in a patient with the potential for subaortic outflow tract obstruction, since mild beta-adrenergic effects may be observed at an epinephrine dose of $1-2 \mu \mathrm{g} \cdot \mathrm{kg}^{-1}$. These effects can include increased heart rate and contractility and mild vascular dilitation. These were not evident in our patient. Kaplan states that these infants would require no sedation due to their quiet nature and decreased activity; ${ }^{*}$ however, our patient was agitated upon arrival in the operating room and was unresponsive to nonpharmacologic measures of comfort, such as allowing the infant to suck on a pacifier or by stroking her cheek.

Low dose ketamine, in combination with the antisialagogue glycopyrrolate, was titrated to calm the infant with minimal or no changes in cardiopulmonary status prior to the placement of the femoral nerve block and to maintain this calm, cooperative state throughout surgery.

In summary, a femoral nerve block and low-dose ketamine sedation were used to perform a diagnostic muscle biopsy in a high-risk infant with a glycogen-storage disease, congestive heart failure, and chronic pulmonary dysfunction. The technique provided excellent anaesthesia for the biopsy without causing detectable alteration in the child's vital signs, skin colour, or capillary refill.

\section{References}

1 Nihill MR, WIlson DS, Hugh-Jones $K$. Generalized glycogenosis type II (Pompe's disease). Arch Dis Child 1970; 45: 122-9.

2 Ellis $F R$. Neuromuscular disease and anaesthesia. Br J Anaesth 1974; 46: 603-12.

3 Winnie AP, Ramamurthy $S$, Durran, $Z$. The inguinal paravascular technic of lumbar plexus anesthesia. The "3-in-1 block". Anesth Analg 1973; 52: 989-96.

4 Wright $B$. A new use for the block-aid monitor. Anesthesiology 1969; 30: 236-7.

5 Harris LC, Powell G, Brown OW III. Pompe's disease. Pediatr Clin North Am 1978; 25: 861-7.

6 Caddell $J L$, Whittemore $R$. Observations of generalized glycogenosis with emphasis on electrocardiographic changes. Pediatrics 1962; 29: 743-63.

7 Hernandez A Jr, Marchesi V. Goldring D, Kissane J, Hartmann $A F$. Cardiac glycogenosis. J Pediatr 1966; 68: 400- 12 .

*Kaplan $R$. Pompe's disease presenting for anesthesia. Anesthesiology Review 1980; 7: 21-8.
8 Ehlers KH, Hagstrom JWC, Lukas PS, Redo SF, Engle MA. Glycogen-storage disease of the myocardium with obstruction to left ventricular outflow. Circulation 1962; 25: 96-109.

9 Rees A, Minhas $K$, Solinger R. Echocardiographic evidence of outflow tract obstruction in Pompe's disease (Glycogen-storage disease of the heart). Am J Cardiol 1976; 37: 1103-6.

10 Hohn AR, Lowe CU, Sokal JE, Lambert EC. Cardiac problems in glycogenosis with specific reference to Pompe's disease. Pediatrics 1965; 35: 313-21.

11 Bharti S, Serratto M, DuBrow/ et al. The conduction system in Pompe's disease. Pediatr Cardiol 1982; $2: 25-32$.

12 Muller $O F$, Bellet $S$, Ertrugrul A. Glycogen-storage disease: report of a case with generalized glycogenosis and review of the literature. Circulation 1961; 23: 261-8.

13 Adams FH, Emmanouilides GC. Heart Disease in Infants, Children, and Adolescents. Baltimore: Williams and Wilkins, 1983.

14 Abajian JC, Mellish RWP, Browne AF, Perkins FM, Lambert $D H$, Mazuzan JE. Spinal anesthesia for surgery in the high-risk infant. Anesth Analg 1984; 63: 359-62.

15 Loubser $P$, Kyoung $S$, Cohen $S$. Adverse effects of spinal anesthesia in a patient with idiopathic hypertrophic subaortic stenosis. Anesthesiology 1984; 60: $228-30$

\section{Résumé}

La conduite anesthésique des enfants atteints de maladie d'entrepasage de glycogène de type lla présente une variété de défis. Une modification du block du nerf fémoral, le block paravasculaire inguinal telle que décrite par Winnie, a été utilisée simultanément avec $l$ 'induction intraveineuse de kétamine afin de fournir l'anesthésie pour une biopsie musculaire diagnostique chez un enfant âgé de 5.5 mois atteinte d'une maladie de Pompe.

Un stimulateur nerveux périphérique était utilisé afin de localiser le nerf fémoral et de provoquer la paresthésie. 